\title{
Biomolecular Detection at ssDNA-Conjugated Nanoparticles by Nano-Impact Electrochemistry
}

Anahita Karimi, Akhtar Hayat and Silvana Andreescu*

Department of Chemistry and Biomolecular Science, Clarkson University, Potsdam, NY, USA

Fax: 3152686610; Tel: 3152682394; *E-mail:eandrees@clarkson.edu

1|P a g e

(C) 2016. This manuscript version is made available under the Elsevier user license http://www.elsevier.com/open-access/userlicense/1.0/ 


\section{Graphical Abstract}
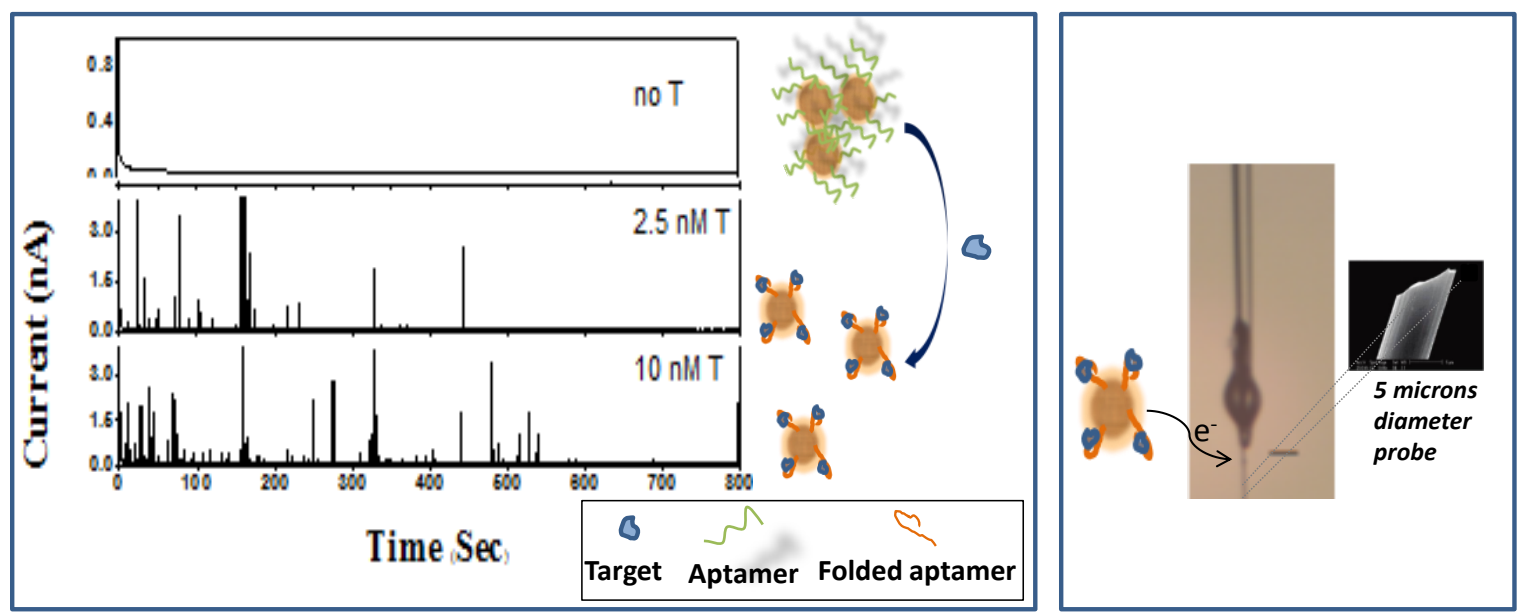


\section{ABSTRACT}

We describe the use of ssDNA functionalized silver nanoparticle (AgNP) probes for quantitative investigation of biorecognition and real time detection of biomolecular targets using nano-impact electrochemistry. The method is based on measurements of the individual collision events between ssDNA aptamer-functionalized AgNPs and a carbon fiber miroelectrode (CFME). Specific binding events of target analyte induced collision frequency changes enabling ultrasensitive detection of the aptamer target in a single step. These changes are assigned to the surface coverage of the NP by the ssDNA aptamers and subsequent conformational changes of the aptamer probe which affect the electron transfer between the NP and the electrode surface. The method enables sensitive and selective detection of ochratoxin A (OTA), chosen here as a model target, with a limit of detection of $0.2 \mathrm{nM}$ and a relative standard deviation of $4.9 \%$. The study provides a means of characterizing bioconjugation of AgNPs with aptamers and assessing biomolecular recognition events with high sensitivity and without the use of exogenous reagents or enzyme amplification steps. This methodology can be broadly applicable to other bioconjugated systems, biosensing and related bioanalytical applications.

Keywords: aptasensor, bioconjugation, impact electrochemistry, target detection, nanoparticles, biomolecular recognition 


\section{INTRODUCTION}

The convergence of nanotechnology and biotechnology has led to the development of hybrid bioanalytical sensing platforms with increased sensitivity, stability and potential for miniaturization and portability in analysis (Katz and Willner 2004). Hybrid nanomaterials that incorporate the selectivity and recognition functions of bioreceptor molecules with the unique size-dependent properties of NPs have proved to be of substantial benefit to the development of bioanalytical assays for clinical diagnosis, biosensing, environmental and food monitoring. A variety of conjugation techniques are available to attach biomolecules to NPs(Ravindran et al. 2013; Sperling and Parak 2010). While widely used, control and characterization of bioconjugation and evaluation of the functionality of the immobilized biomolecule with adequate sensitivity remain a challenge. Attachment of biomolecules can affect the structure of the biomolecule (Vertegel et al. 2004) while excessive coverage can reduce its activity due to steric crowding (Demers et al. 2000). The ability to investigate biomolecular recognition events at the surface of single particles is important for the rational design of molecular recognition probes for a variety of applications including therapeutics, cell tracking, drug delivery systems, biosensing and for the development of sensitive bioanalytical assays.

Among bioconjugated NPs, aptamer functionalized NPs that use artificial single stranded oligonucleotides have gained popularity in recent years due to their high stability and ability to recognize and bind to a variety of targets with high specificity and selectivity (McConnell et al. 2014). When bound to a target, aptamers undergo significant conformational changes (Fan et al. 2003). The folding/unfolding of aptamers is essential for their recognition ability. Commonly used methods to characterize NP-aptamer bioconjugates include UV-Vis and fluorescence spectroscopy (Yu and Irudayaraj 2006) that rely on bulk measurements of the conjugate system. Achieving accurate quantitative data on the binding using these methods is difficult because of relatively small changes in the optical spectra, background interferences and spectral overlap. Combining UV-Vis spectroscopy and chemometrics by introduction of multivariate curve resolution enhanced the resolving power of traditional UV-Vis spectroscopy (Wang and $\mathrm{Ni}$ 2014). Incorporating a fluorescent label at the terminus of the DNA sequence enabled quantitation by fluorescence spectroscopy through displacement of the surface-bound strands (Demers et al. 2000). Enzyme hydrolysis of fluorescent-labeled biomolecules, stepwise 
photobleaching of organic fluorophores, single particle fluorescence resonance energy transfer (spFRET), adsorption isotherms, and gel electrophoresis have also been used to assess the coupling of biomolecules to NPs(Pons et al. 2006). In this work, we describe a novel single step and sensitive electrochemical approach for the characterization of aptamer conjugated NPs and detection of biomolecular targets using nano-impact electrochemistry.

The rapidly growing field of nano-impact electrochemistry involving measurement of single particle collision events with an electrode surface has made a significant impact on the study of NP systems. Nano-impact electrochemistry has demonstrated promise for the detection and characterization of a variety of NPs including metals, metal oxides and organic particles(Cheng et al. 2013; Sardesai et al. 2013; Xiao and Bard 2007), and could be of use for the characterization of NPs in a variety of environments(Ozel et al. 2014; Sardesai et al. 2013). Starting from the pioneering work of Bard group(Xiao and Bard 2007), several groups including Stevenson (Dasari et al. 2013), Crooks (Alligrant et al. 2015), Compton (Cheng and Compton 2014; Rees et al. 2012; Stuart et al. 2012), Zhang (Guo et al. 2014), Alpuche-Aviles (Fernando et al. 2013), and a few others have all made significant contributions to the field of nano-impact collision electrochemistry. Previous researches demonstrated the ability of the method to evaluate the catalytic properties, size distribution, concentration, aggregation degree and adsorption processes at NPs (Cheng and Compton 2014; Rees et al. 2011; Zhou et al. 2010). More recently, additional capabilities have been demonstrated to measure E.Coli bacteria decorated with AgNPs (Sepunaru et al. 2015), NPs tagged with electroactive molecules (Zhou et al. 2012), analysis of DNA hybridization by indirect measurement of hydrazine oxidation (Kwon and Bard 2012) and for detection of viruses by antibodies modified polystyrene beads and singleprotein and DNA macromolecule (Dick et al. 2015). The group of Crooks (Alligrant et al. 2015; Alligrant et al. 2013) has recently reported on the electrocatalytic amplification on single collisionsof PtNPs using DNA-modified surfaces. Despite significant progress on the development of this method, studies to investigate the capability of this method in the bioanalytical and biosensing field for detection of biomolecular targets are limited.

Here we investigate the use of NP collision electrochemistry as a detection technique for characterizing bioreceptor NP conjugates and quantitatively measuring target analytes using aptamer-modified AgNPs. As a proof of concept, we developed the method for detection of OTA. OTA is one of the most-abundant food-contaminating mycotoxins produced by fungi species 
such as aspergillus and penicillium. OTA is classified as a potential carcinogen (group 2B) for humans (Ha 2015). Several types of electrochemical aptamer-based bioassays have been developed using target-induced structure switching mode by probing the electron-transfer at aptamer modified electrodes in various configurations (Bulbul et al. 2015a, b). Most of these methods involve sensitive enzyme labels, soluble redox mediators, extensive electrode modification or multistep procedures to achieve the required detection limits for practical applications. As opposed to previous studies, here we describe a single step procedure that employs a bare CFME and AgNPs modified with aptamers and uses NP collision electrochemistry to characterize the transient current generated upon the individual impact of aptamer-modified AgNPs with the electrode. Changes in the current response from collisions of conjugated aptamer-AgNPs enable real time quantitative detection of the aptamer target with high sensitivity. The method can be particularly useful in fundamental studies of binding mechanisms of aptamer-NP conjugates, and in biosensing/bioanalytical applications that require recognition and ultrasensitive detection of biomolecular targets.

\section{Materials and methods}

\subsection{Reagents and chemicals.}

5'-amino-modified aptamer strands were provided by Eurogentec (North America Inc.). The sequences for the given strand is ( 5 ' $\mathrm{NH}_{2}$-GAT-CGG-GTG-TGG-GTG-GCG-TAA-AGG-GAGCAT-CGG-ACA 3'). Phosphate buffer (PB) solution, 0.1M, pH 7.4 was prepared from sodium phosphate (monobasic and dibasic) (Fisher Sci.). The binding buffer (BB) was prepared from 1 $\mathrm{mM} \mathrm{MgCl} 2,140 \mathrm{mM} \mathrm{NaCl}, 2.7 \mathrm{mM} \mathrm{KCl}$. OTA (from A. ochraceous) and Ochratoxin B (OTB) were first dissolved in ethanol and then diluted in binding buffer. OTA and OTB were supplied by Sigma-Aldrich. Carbon fibers were purchased from WPI (World Precision Instruments Inc.). Silver conductive epoxy was obtained from MG Chemicals. Non-conductive epoxy was purchased from Devcon. Distilled, deionized water (Millipore, Direct-Q System) with a resistivity of $18.2 \Omega \mathrm{cm}$ was used. Citrate capped silver nanoparticles (AgNPs) were generously provided by Dr. Dan Goia, Clarkson University.

\subsection{Instrumentation.}

6 | P a g e 
Electrochemical experiments were performed using a $\mathrm{CH}$ Instrument analyzer $(\mathrm{CH}$ Instruments Inc.) (Austin, USA). All experiments were carried out using a conventional electrochemical cell with cylindrical carbon microelectrodes as the working electrode, $\mathrm{Ag} / \mathrm{AgCl} / 3 \mathrm{M} \mathrm{KCl}$ as reference electrode and a platinum wire as counter electrode. All potentials were referred to the $\mathrm{Ag} / \mathrm{AgCl}$ reference. UV-Vis studies were performed with a Shimadzu PC-2401 spectrophotometer (Kyoto, Japan). Transmission Electron Microscopy (TEM) experiments were performed using a JEOL 2010 High Resolution Scanning Transmission Electron Microscope (HRTEM) (Peabody, USA). Samples were dried before measurements. Particle sizing and zeta potential measurements were carried out using a Brookhaven Instruments Zeta PALS analyzer (Holtsville, USA).

\subsection{Preparation of cylindrical carbon fiber microelectrodes.}

Single CFME with a diameter of $\sim 2.5 \mu \mathrm{m}$ were fabricated from individual carbon fibers from WPI. A single carbon fiber was glued to a copper wire with a conductive silver epoxy paste and aspirated into a pulled glass capillary tube. The upper end of the capillary tube was sealed with a non-conductive epoxy resin and cured at $100^{\circ} \mathrm{C}$ for $10 \mathrm{~min}$. The capillary tube with the connecting wire was pulled using a Narishige PP-83 microelectrode puller. The length of the fiber extending from glass was fixed to $\sim 1 \mathrm{~mm}$ from the glass seal using a scalpel. Microelectrodes were immersed in isopropanol for $10 \mathrm{~min}$ to clean the fibers and air dried.Before experiments the electrodes were characterized by cyclic voltammetry (CV) using the potassium ferricynianide/ferrocyanide redox couple to determine the surface area and ensure batch-to-batch reproduciblility.

2.4. Preparation of AgNP aptasensor. Prior to modification, the aptamer solution was heated at $95^{\circ} \mathrm{C}$ for $5 \mathrm{~min}$ and subsequently left at room temperature for $3 \mathrm{~min}$. Citrate capped AgNPs were used as support for the immobilization of the 5' amino aptamer through the amino group. The immobilization protocol consisted of the following steps: 1) $10 \mathrm{mg} \mathrm{AgNP}$ were dispersed in $1 \mathrm{ml}$ PB and sonicated. 2) The dispersion was centrifuged and $1 \mathrm{ml}$ 1-ethyl-3-(3dimethylaminopropyl)carbodiimide (EDC) at a concentration of $15 \mathrm{mg} / \mathrm{ml}$ was added to the dispersion to activate the carboxylic acid groups of the citrate capped NPs. 3) The mixture was incubated using a shaker for $3 \mathrm{~h}$ and then centrifuged and washed twice with $\mathrm{PB}$ to remove 
excess EDC. 4) The activated particles were re-suspended in $1 \mathrm{ml} \mathrm{PB}$. 5) $100 \mu \mathrm{L}$ of aptamer solution $(0.4 \mathrm{nM}$ to $0.25 \mu \mathrm{M}$ prepared in $\mathrm{BB})$ was added to $4 \mathrm{ul}$ of activated NP dispersion and incubated for 5 hours with slow rotation. After the $5 \mathrm{hrs}$ incubation, the aptamer-AgNP conjugates were separated by centrifugation for $10 \mathrm{~min}$ at $13000 \mathrm{rpm}$ and washed twice with 0.1 M PB. The conjugates were then re-suspended in $500 \mu \mathrm{L} \mathrm{PB}$.

2.5. Electrochemistry experiments. The electrochemical behaviour of the AgNPs in PB after each modification step was studied using CV and nano-impact electrochemistry. CVs were obtained in the potential range from -0.7 to $0.7 \mathrm{~V}$ at a $100 \mathrm{mV} / \mathrm{s}$ scan rate in $\mathrm{PB}$ at $\mathrm{pH} 7.4$. For nano-impact experiments, the cylindrical CFME was potentiostated at $+0.5 \mathrm{~V}$ to measure $\mathrm{Ag}$ oxidation and generate the oxidative spikes and collision profiles. Detection of OTA was also investigated using electrochemistry. $100 \mu \mathrm{L}$ of OTA (at concentrations ranging from 0.01 to 10 $\mathrm{nM}$ ) was added to $4 \mu \mathrm{L}$ aptamer-AgNP conjugates and incubated for 3 hrs. After that the NPs were dispersed in PB and injected to the electrochemical cell. All experiments reported in this work have been repeated at least six times with independently prepared electrodes. The standard deviation on calibration curves represents electrochemical recordings for $n=6$ replicates.

\section{RESULTS AND DISCUSSION}

\subsection{Electrochemical collision of aptamer binding.}

AgNPs and ssDNA aptamers against OTA were selected in this work as a model system. Electrochemical collision signals at the surface of a CFME surface were used to probe electron transfer changes at the AgNP surface upon conjugation with aptamer. A cylindrical form CFME with a radius $2.5 \mu \mathrm{m}$ and a length of $1 \mathrm{~mm}$ was used in all experiments (Ellison et al. 2014). To perform measurements, the CFME was placed in 0.1 $\mathrm{M} \mathrm{PB}$ at a $\mathrm{pH}$ of 7.4 containing aptamer-conjugated AgNPs.Attachment of aptamer to AgNPs was achieved by covalent bonding between the carboxylic group of the capping agent on the surface of NPs and the amine $5^{6}$ terminus of the aptamer using EDC as described in Supporting Information (SI) (Scheme S-1). For quantification purposes only spikes with intensity values above $0.2 \mathrm{nA}$ were considered for counting. In the absence of 
aptamer, the chronoamperometric collision profile shows anodic spikes corresponding to the oxidation of AgNPs. Modification of the AgNPs with aptamers induces a significant decrease in the collision frequency. The number of spikes decreases proportionally with the amount of aptamer (Figure 1). This decrease and the linear dependency indicate surface coverage and insulation of the electrochemically accessible surface area of AgNPs by aptamers, hampering the electron transfer. The Bard and Crooks groups (Alligrant et al. 2015; Xiao et al. 2009) reported electron transfer blockage at DNA-modified NPs for DNA strands longer than 25mer (Alligrant et al. 2015). Here, we used a DNA strand of 36 mer with a length of $12.24 \mathrm{~nm}$, which provided sufficient length to block charge transfer between AgNPs and the CFME. More than $60 \%$ of the DNA strand comprises nitrogencontaining bases ( $\mathrm{C}$ and $\mathrm{G}$ ). The $\mathrm{C}$ and $\mathrm{G}$ bases of covalently bound aptamer can also attach to the unoccupied Ag surface due to strong interaction of DNA bases with NPs (Basu et al. 2008; Petty et al. 2004) resulting in formation of DNA-bridged NP aggregates (Figure 1). The decrease in number of spikes can also be explained by a lower diffusion coefficient of aggregated AgNP that are known to reduce collision frequency (Ellison et al. 2013).Fluorescence spectrometry measurements used as a complementary technique to determine the aptamer coverage on AgNPs modified with FAM (Fluorescein) attached to 3' end of strand (Figure S-1, Table S-1) show an increase in fluorescence intensity until it reaches a plateau at a concentration of $0.63 \mu \mathrm{M}$. The difference in concentration can be attributed to difference in the detection modality based on Ag oxidation due to NP insulation by the aptamer, in the case of electrochemistry, versus the measurement of the FAM tagged on ss-DNA for fluorescence measurement.

In further experiments, we have performed analysis of oxidative impact transients to monitor the charge passed per impact transient and plotting the size histogram of the aptamer conjugated NPs using Origin software(Rees et al. 2011; Stuart et al. 2012). The charge passed per impact transient, $\mathrm{Q}$, corresponding to the number of atoms involved in collision was used to calculate the NP size using the equation (1):

$\mathrm{R}_{\mathrm{NP}}=\sqrt[3]{\frac{3 Q A_{r}}{4 \pi F \rho}}$

in which $R_{N P}$ is the NP radius, $Q$ is the total charge passed under a single spike, $A_{r}$ is the atomic mass $\left(107.9 \mathrm{~g} \mathrm{~mol}^{-1}\right), \mathrm{F}$ is the Faraday constant and $\rho$ is the density of $\mathrm{Ag}$ 
$\left(10.5 \times 106 \mathrm{~g} \mathrm{~m}^{-3}\right)$. Figure 2 shows the size distribution of bare and aptamer conjugated AgNPs obtained from electrochemical sizing of AgNPs after conjugation. The radius of bare NPs calculated from the collision profile showed a particle size distribution with a maximum size of $\sim 33 \mathrm{~nm}$. Conjugation with different amounts of aptamer results in increased NP size which can be related to surface coverage by the aptamer and formation of NP clusters. It is known that DNA oligonucleotides have high affinity for Ag ions through the heterocyclic bases which facilitates DNA adsorption and formation of Ag nanoclusters (Petty et al. 2004). Interparticle DNA base bridging through affinity for Ag, as well as Van der Waals attraction forces and DNA base pairing may induce aggregation of the ssDNA-AgNPs generating an overall increase in conjugate size (Zhao et al. 2008).Indeed, conjugation of AgNPs with aptamer results in a gradual shift in size from $\sim 33 \mathrm{~nm}$ to $\sim 50 \mathrm{~nm}$, reaching a maximum cluster size of 50-100 nm for 0.01, 0.03 and 0.12 $\mu \mathrm{M}$ aptamer concentrations respectively. Higher aptamer concentrations caused complete aggregation and insulation. As a result, no collision signals were observed. Since the number of collision events correlates with the electrochemically exposed surface of AgNPsthis method can be used to study adsorption and size dependent nanocluster formation of aptamer functionalized NPs.

\subsection{Validation of NP collision signals.}

In order to confirm the origin of the collision spikes and the surface attachment, UV-Vis and fluorescence spectroscopy and CV were used to demonstrate the electrochemical oxidation and determine changes in surface coverage in bulk NP dispersion before and after conjugation (Figure 3). CVs of AgNPs dispersion upon addition of aptamer show a gradual decrease of the oxidative AgNP peak (FigureS-2). The same trend was observed in the UV-Vis spectra. Following the addition of $0.25 \mu \mathrm{M}$ aptamer, the surface plasmon absorption band of AgNPs at $400 \mathrm{~nm}$ disappeared. This correlates with the absence of the $\mathrm{Ag}$ peak in the $\mathrm{CV}$ and the lack of collision signals at this concentration. Therefore both methods confirm a concentration-dependent change in the surface and oxidation behavior of AgNPs conjugated with aptamer. The ssDNA aptamer coverage determined by fluorescence spectrometry measurements using AgNPs modified with FAM (Fluorescein) (Table S-1) also show a concentration-dependent increase in fluorescence intensity. TEM and dynamic 
light scattering (DLS) were further employed to confirm the NP size predicted by single particle collision experiments. Bare AgNPs showed an average particle size measured by DLS of $40( \pm 3.9) \mathrm{nm}$ with a negative zeta potential of $-41.5( \pm 2.13) \mathrm{mV}$. Upon addition of aptamer $(0.25 \mu \mathrm{M})$ the size increased to $242.4( \pm 8.3) \mathrm{nm}$ indicating an aggregation effect. The TEM image confirms an average particle size of bare AgNPs between 30 and $40 \mathrm{~nm}$ (Figure4). After the addition of aptamer, the conjugated NPs form visible clusters as predicted by the collision, $\mathrm{CV}$ and $\mathrm{UV}$-Vis tests.

\subsection{Analytical performance for detection of aptamer targets.}

In the next set of experiments, the aptamer conjugated AgNPs were used as detection probes for the target analyte. As a proof-of-concept, we have developed a method for the detection of OTA. Spectroscopy results show that addition of OTA to the aptamerconjugated AgNPs generated a concentration dependent increase in the AgNP absorption peak at $400 \mathrm{~nm}$ in the UV-Vis spectra (Figure 3) and a gradual increase in the $\mathrm{Ag}$ oxidation peak in the CV (Figure S-3). We hypothesize that as the target binds to the immobilized aptamer, the aptamer changes conformation from a loose random coil to a folded structure destabilizing the electrostatic interaction involving the nitrogencontaining bases ( $\mathrm{C}$ and $\mathrm{G}$ ) that are responsible for the DNA-assisted interparticle association. A compact folded structure of ss-DNA grafted on NPs can lead to steric stabilization andinduced de-aggregation(Zhao et al. 2008).Therefore, aptamer folding alters the electron transfer tunnelling distance between the aptamer-target-AgNP conjugates and the CFME. Indeed, the TEM image (Figure 4) shows complete deaggregation of the AgNPs-aptamer clusters upon target addition. The DLS measurements indicate a particle size of $42.8( \pm 4.5) \mathrm{nm}$ with a negative zeta potential of $-53.8( \pm 3.45)$ $\mathrm{mV}$. We have further investigated the suitability of nano-impact electrochemistry to characterize aptamer-target binding interactions and to quantitatively determine target molecules, OTA. The collision spectra do not show any significant spike for the aptamerconjugated AgNPs, in the absence of target. Upon addition of OTA, a detectable current transient is observed when the aptamer-modified AgNPs hit the electrode. When the aptamer-Ag nanoclusters are incubated with OTA and injected to the electrochemical cell, the change in conformation of the immobilized aptamer upon target binding, and the 
associated de-aggregation effect facilitates electron transfer between the AgNP surface and the CFME which is clearly reflected in the gradual increase in collision frequency. This appearance of oxidative spikes indicates collision of more individual AgNPs with the CFME surface (Figure 5). This evidence suggests that the electrochemically active surface of AgNP-aptamer clusters become more accessible in the presence of target molecules. The size distribution of AgNPs from the collision profile indicates a gradual decrease in the aggregate size with average values of $\sim 50$ to $30 \mathrm{~nm}$ for OTA concentrations ranging from 0.07 to $10 \mathrm{nM}$ (Figure S-4). Since spike frequency varies linearly with the concentration of OTA the method can be used as a quantitative tool for the determination of target molecules. The lowest concentration of OTA that can be determined by this method is $0.2 \mathrm{nM}$, with a relative standard deviation of $4.9 \%$. This

limit is below the threshold set by the World Health Organization for OTA (Denli and Perez 2010). Several works have reported lower detection limits for OTA with other electrochemical aptasensing platforms. These methods involved addition of reagents, enzyme amplifiers, redox mediators and multistep procedures (Bonel et al. 2011; Chrouda et al. 2015; Perrotta et al. 2012).

\subsection{Selectivity study.}

To evaluate selectivity of this method, we have conducted experiments using a control molecule, ochratoxin B (OTB), a structurally similar compound with significantly lower or no binding affinity for the OTA aptamer (Cruz-Aguado and Penner 2008). As expected, the addition of OTB did not induce any noticeable change in CV and collision profile (Figure S-5 and Figure 6). This evidence suggests that the process is highly specific to the binding of target molecule. Therefore the specificity of this method is confirmed. As compared to previously developed aptasensors, the method reported here is simpler, provides high sensitivity and does not involve enzyme labels, addition of exogenous reagents or elaborate amplification steps. Moreover, the concept can be used for detection of other bimolecular targets. The method can also be extended to the study of other types of biomolecular interactions involving conductive NPs.

\section{Conclusion}


In summary, we have demonstrated the possibility to detect biomolecular aptamer targets in a single step using nano-impact electrochemistry. This methodology allows characterization of bioconjugation and biomolecular recognition at aptamerfunctionalized NP probes. The technique can lead to advancements in the analytical chemistry field and developments of methodology for detection and fundamental study of biorecognition and molecular diagnosis probes in the biomedical, biosensing and biotechnology fields. Our results demonstrate that: 1) NP collision electrochemistry can be used as an analytical quantification method to assess binding of aptamers to individual NPs, 2) the collision profile provides a real-time measure of the aptamer target binding at NP surfaces, 3) the collision frequency varies proportionally with the concentration of surface bound aptamers and target molecules, and 4) the method provides sensitive detection of aptamer targets using a single step procedure, demonstrated here with OTA as model. These findings indicate that nano-impact electrochemistry can serve as a powerful technique for examining NP-aptamer interactions and for detecting molecular targets. The proposed strategy may find use in biomolecular recognition studies of aptamer functionalized NPs for bio-detection and therapeutic applications.

\section{Acknowledgements.}

This material is based upon work supported by the National Science Foundation under Grant No. 1610281. Any opinions, findings, and conclusions or recommendations expressed in this material are those of the author(s) and do not necessarily reflect the views of the National Science Foundation.

\section{References}

Alligrant, T.M., Dasari, R., Stevenson, K.J., Crooks, R.M., 2015. Langmuir. 31, 11724-11733. Alligrant, T.M., Nettleton, E.G., Crooks, R.M., 2013. Lab on a Chip. 13, 349-354. Basu, S., Jana, S., Pande, S., Pal, T., 2008. J. Colloid Interface Sci. 321, 288-293. 
Bonel, L., Vidal, J.C., Duato, P., Castillo, J.R., 2011. Biosensors and Bioelectronics. 26, 32543259.

Bulbul, G., Hayat, A., Andreescu, S., 2015a. Nanoscale. 7, 13230-13238.

Bulbul, G., Hayat, A., Andreescu, S., 2015b. Sensors. 15, 30736-30758.

Cheng, W., Compton, R.G., 2014. TrAC Trends in Analytical Chemistry. 58, 79-89.

Cheng, W., Zhou, X.-F., Compton, R.G., 2013. Angewandte Chemie International Edition. 52, 12980-12982.

Chrouda, A., Sbartai, A., Baraket, A., Renaud, L., Maaref, A., Jaffrezic-Renault, N., 2015. Anal. Biochem. 488, 36-44.

Cruz-Aguado, J.A., Penner, G., 2008. J. Agric. Food Chem. 56, 10456-10461.

Dasari, R., Robinson, D.A., Stevenson, K.J., 2013. J. Am. Chem. Soc. 135, 570-573.

Demers, L.M., Mirkin, C.A., Mucic, R.C., Reynolds, R.A., Letsinger, R.L., Elghanian, R., Viswanadham, G., 2000. Anal. Chem. 72, 5535-5541.

Denli, M., Perez, J.F., 2010. Toxins. 2, 1065-1077.

Dick, J.E., Hilterbrand, A.T., Boika, A., Upton, J.W., Bard, A.J., 2015. Proceedings of the

National Academy of Sciences. 112, 5303-5308.

Ellison, J., Batchelor-McAuley, C., Tschulik, K., Compton, R.G., 2014. Sensors and Actuators

B: Chemical. 200, 47-52.

Ellison, J., Tschulik, K., Stuart, E.J.E., Jurkschat, K., Omanović, D., Uhlemann, M., Crossley, A., Compton, R.G., 2013. ChemistryOpen. 2, 69-75.

Fan, C.H., Plaxco, K.W., Heeger, A.J., 2003. Proc. Natl. Acad. Sci. U. S. A. 100, 9134-9137.

Fernando, A., Parajuli, S., Alpuche-Aviles, M.A., 2013. J. Am. Chem. Soc. 135, 10894-10897.

Guo, Z., Percival, S.J., Zhang, B., 2014. J. Am. Chem. Soc. 136, 8879-8882.

Ha, T.H., 2015. Toxins. 7, 5276-5300.

Katz, E., Willner, I., 2004. Angewandte Chemie International Edition. 43, 6042-6108.

Kwon, S.J., Bard, A.J., 2012. J. Am. Chem. Soc. 134, 10777-10779.

McConnell, E.M., Holahan, M.R., DeRosa, M.C., 2014. Nucleic Acid Ther. 24, 388-404.

Ozel, R.E., Liu, X.B., Alkasir, R.S.J., Andreescu, S., 2014. Trac-Trend Anal Chem. 59, 112-120.

Perrotta, P.R., Arévalo, F.J., Vettorazzi, N.R., Zón, M.A., Fernández, H., 2012. Sensors and

Actuators B: Chemical. 162, 327-333.

Petty, J.T., Zheng, J., Hud, N.V., Dickson, R.M., 2004. Journal of the American Chemical

Society. 126, 5207-5212.

Pons, T., Medintz, I.L., Wang, X., English, D.S., Mattoussi, H., 2006. J. Am. Chem. Soc. 128, 15324-15331.

Ravindran, A., Chandran, P., Khan, S.S., 2013. Colloid Surface B. 105, 342-352.

Rees, N.V., Zhou, Y.-G., Compton, R.G., 2011. ChemPhysChem. 12, 1645-1647.

Rees, N.V., Zhou, Y.-G., Compton, R.G., 2012. Chem. Phys. Lett. 525-526, 69-71.

Sardesai, N.P., Andreescu, D., Andreescu, S., 2013. Journal of the American Chemical Society. 135, 16770-16773.

Sepunaru, L., Tschulik, K., Batchelor-McAuley, C., Gavish, R., Compton, R.G., 2015.

Biomaterials Science.

Sperling, R.A., Parak, W.J., 2010. Phil. Trans. R. Soc. A. 368, 1333-1383.

Stuart, E.J.E., Zhou, Y.-G., Rees, N.V., Compton, R.G., 2012. RSC Advances. 2, 6879-6884.

Vertegel, A.A., Siegel, R.W., Dordick, J.S., 2004. Langmuir. 20, 6800-6807.

Wang, Y., Ni, Y., 2014. Talanta. 119, 320-330.

Xiao, X., Bard, A.J., 2007. J. Am. Chem. Soc. 129, 9610-9612. 
Xiao, X., Pan, S., Jang, J.S., Fan, F.-R.F., Bard, A.J., 2009. The Journal of Physical Chemistry C. 113, 14978-14982.

Yu, C., Irudayaraj, J., 2006. Anal. Chem. 79, 572-579.

Zhao, W., Chiuman, W., Lam, J.C., McManus, S.A., Chen, W., Cui, Y., Pelton, R., Brook, M.A., Li, Y., 2008. J. Am. Chem. Soc. 130, 3610-3618.

Zhou, H., Fan, F.R.F., Bard, A.J., 2010. Journal of Physical Chemistry Letters. 1, 2671-2674.

Zhou, Y.-G., Rees, N.V., Compton, R.G., 2012. Chem. Commun. 48, 2510-2512. 


\section{Figure captions}

Figure 1. (A) Chronomperometric collision profiles of AgNPs $(0.01 \mathrm{mg} / \mathrm{ml})$ before and after conjugation with varying concentrations of aptamer. Anodic current spikes show oxidation of AgNPs colliding to CFME. Experiments were performed in phosphate buffer $(\mathrm{pH}=7.4)$. (B) The CFME potential was $0.5 \mathrm{~V}$ vs. $\mathrm{Ag} / \mathrm{AgCl}$. Calibration curve showing the number of collisions versus concentration of aptamer.

Figure 2. Illustrative size distribution of AgNPs electrochemical collision signals before (A) and after conjugation with (B) $0.01 \mu \mathrm{M},(\mathrm{C}) 0.03 \mu \mathrm{M}$ and (D) $0.12 \mu \mathrm{M}$ aptamer.

Figure 3. (A) UV-vis absorption spectra of AgNP dispersion before and after conjugation with $0.25 \mu \mathrm{M}$ aptamer, and after addition of target molecule (OTA, $30 \mathrm{nM}$ ). (B) Increase in the AgNPs absorption band with increasing concentrations of OTA from 0 to $30 \mathrm{nM}$.

Figure 4. TEM images of (1) bare AgNPs (2) conjugated with $0.025 \mu \mathrm{M}$ aptamer and (3) after addition of target molecule (OTA, $30 \mathrm{nM})$

Figure 5. (A) Representative chronoamperometric response for a CFME potentiostated at $0.5 \mathrm{~V}$ vs. $\mathrm{Ag} / \mathrm{AgCl}$ in $0.1 \mathrm{M} \mathrm{PB}$ with AgNPs-aptamer in the presence of different concentration of OTA. (B) Calibration curve and linearity range showing a linear relationship between the number of collisions and OTA concentration.

Figure 6. Target selectivity: chronamperometric collision profile of aptamer-AgNP conjugates in the presence of OTB $(30 \mathrm{nM})$ as a control target molecule. 

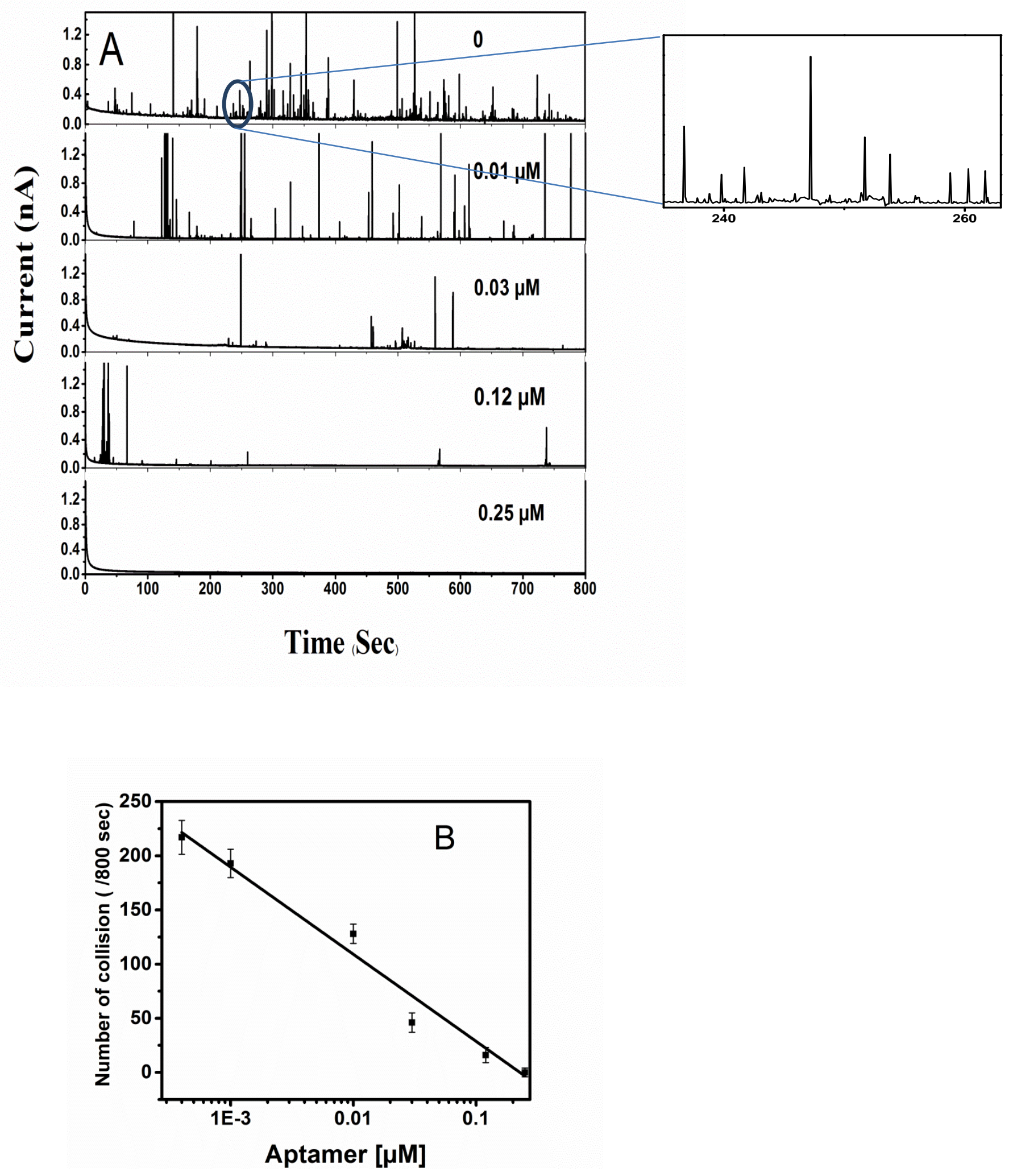

Figure 1 

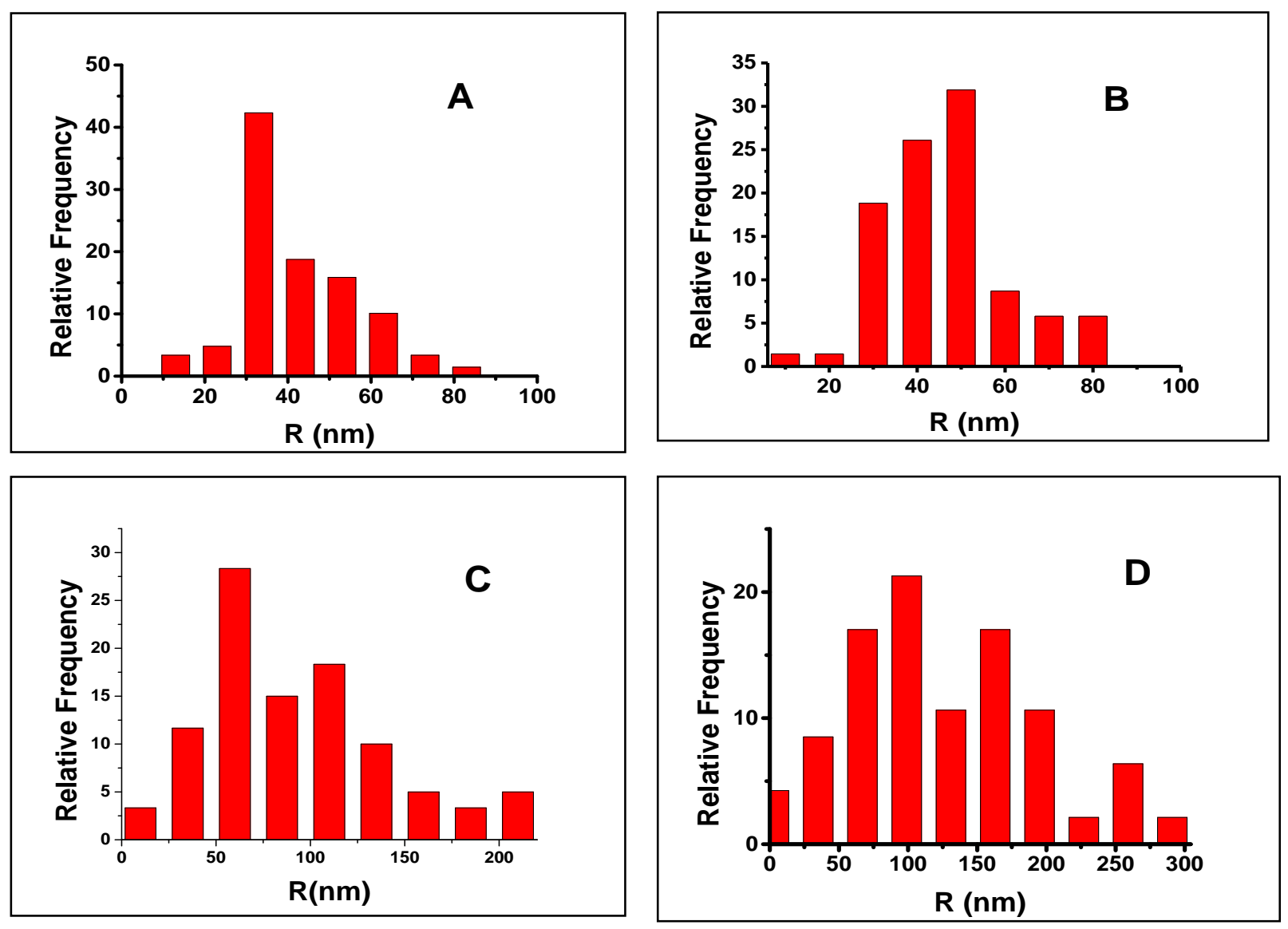

Figure 2 

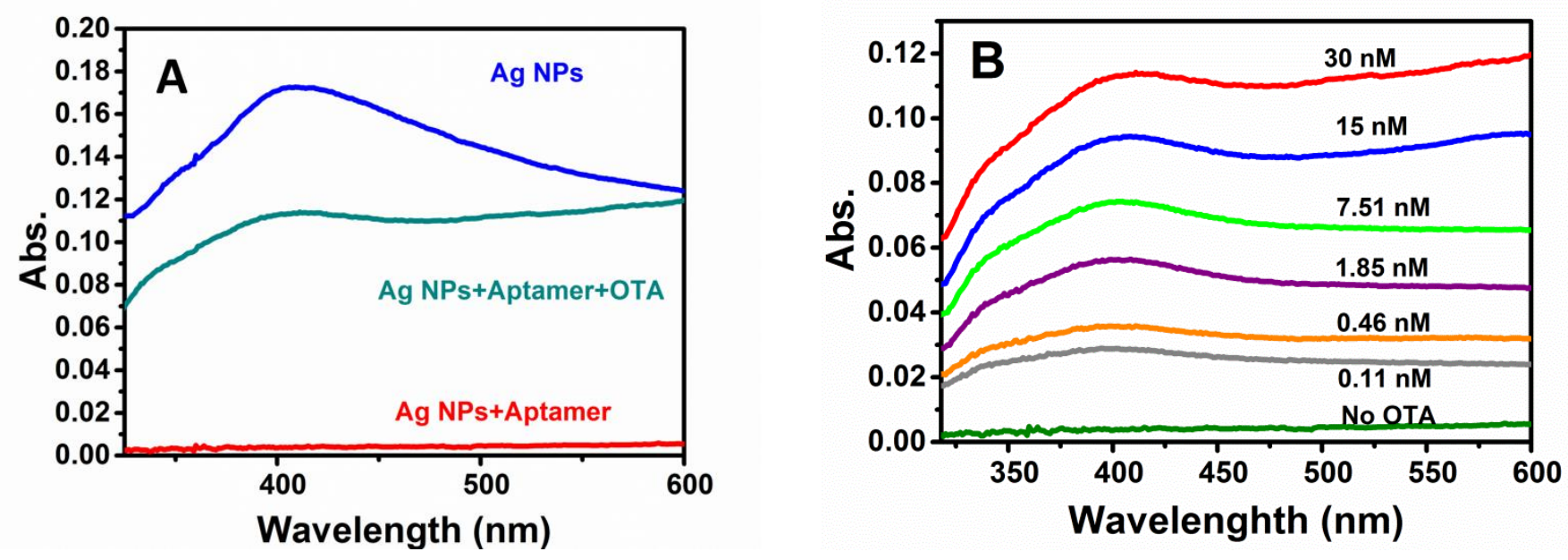

Figure 3 


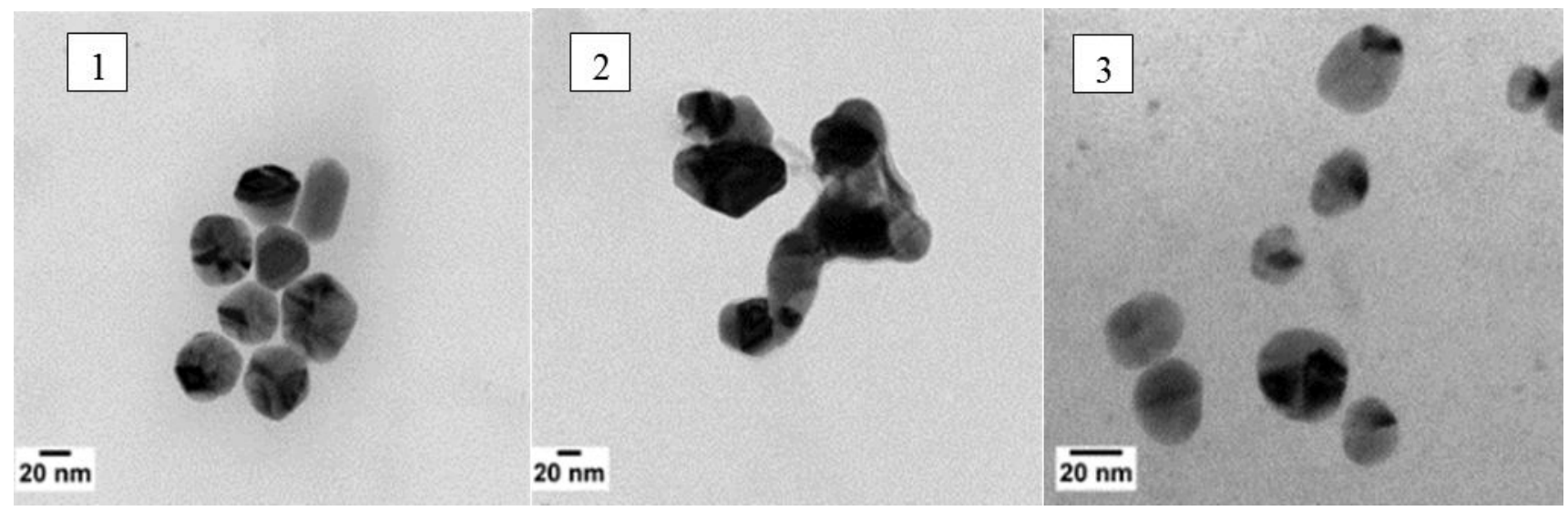

Figure 4 

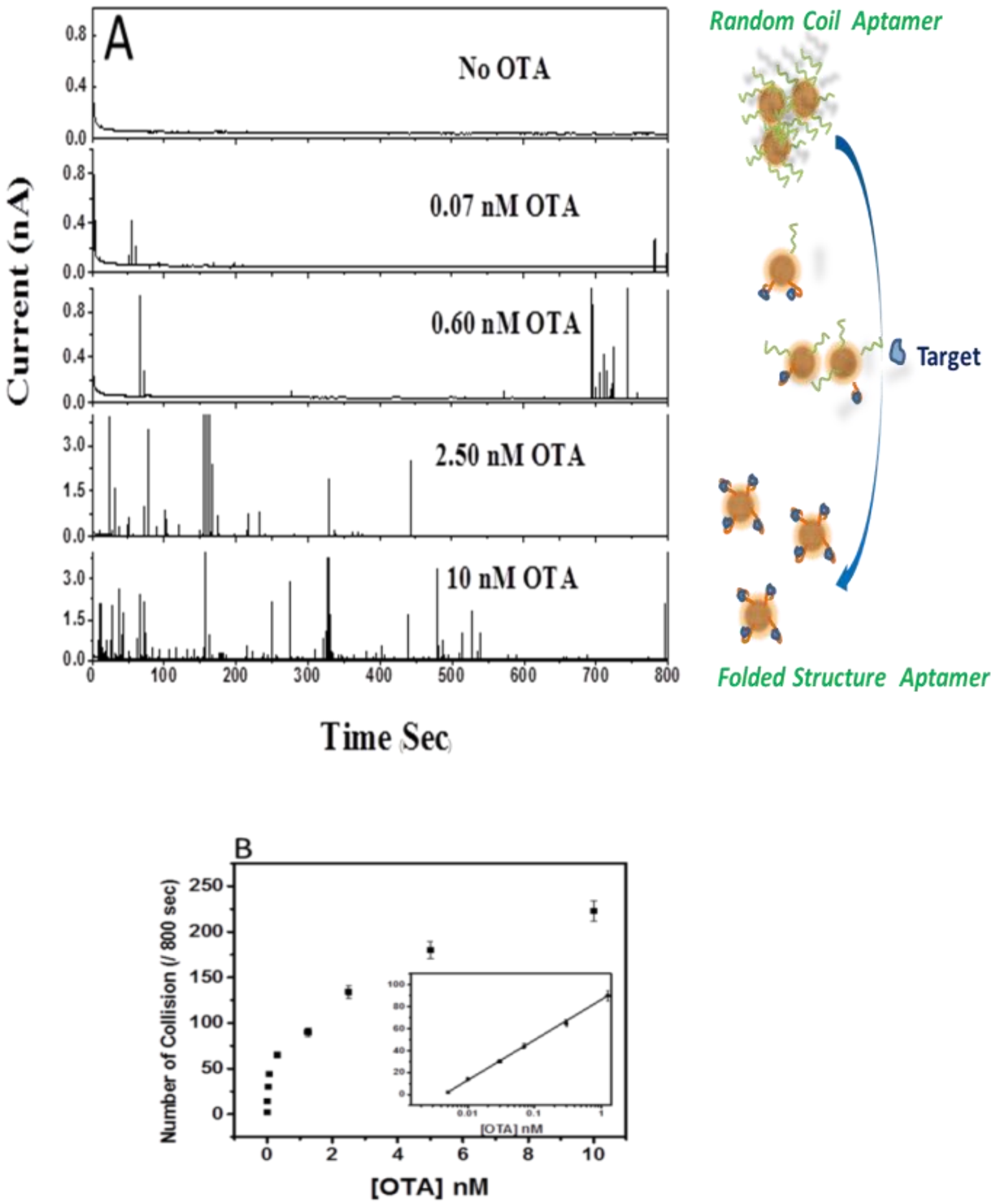

Figure 5 


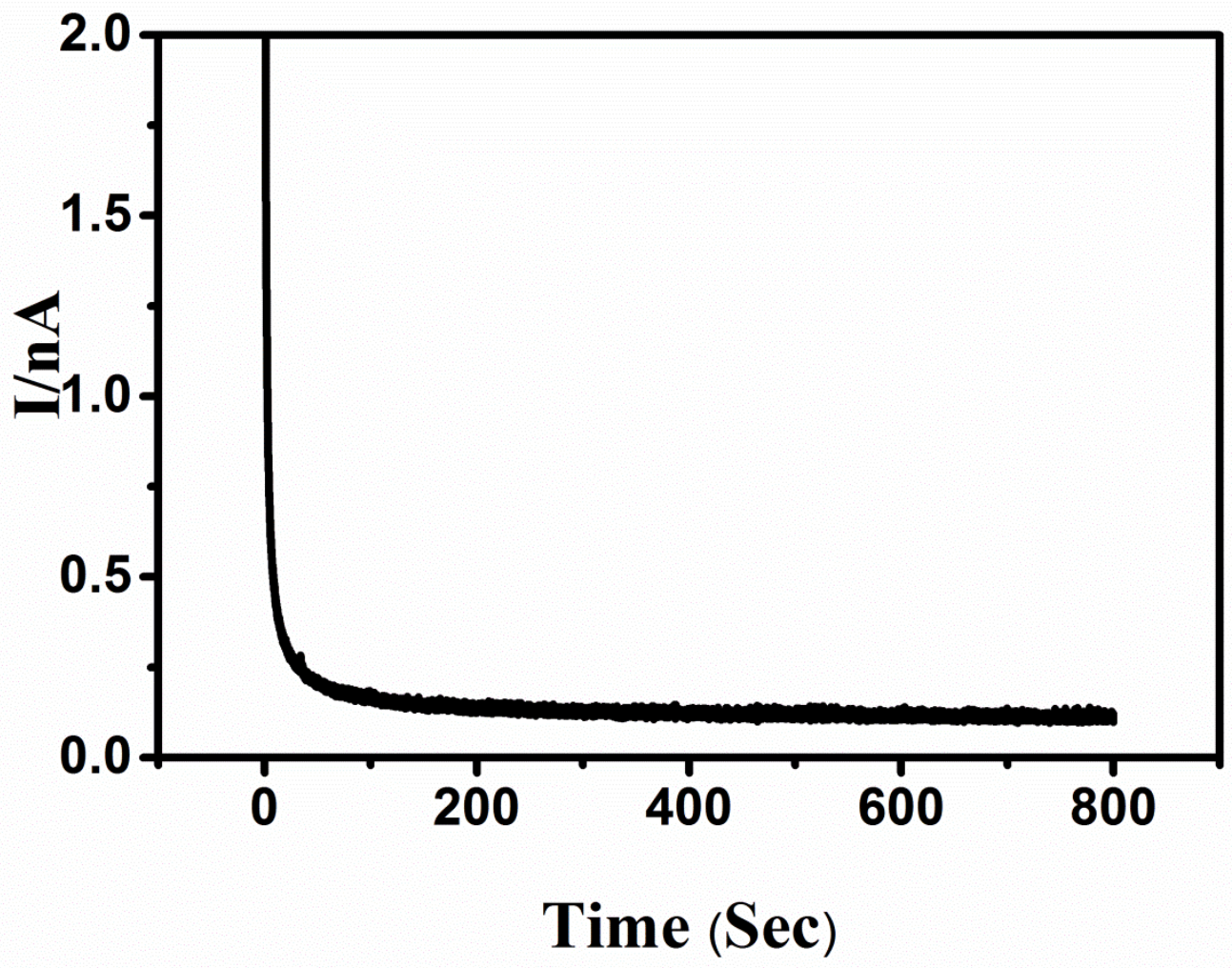

Figure 6 